\title{
Parent, Teacher and Observational Reports of Emotional and Behavioral Problems in Young Autistic Children
}

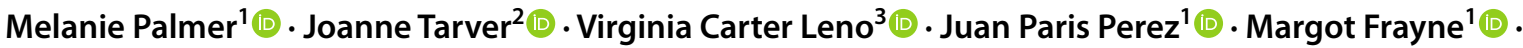

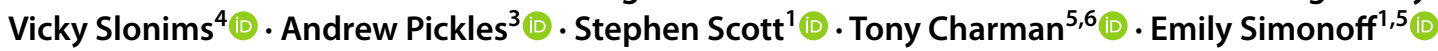

Accepted: 21 December 2021 / Published online: 13 January 2022

(c) The Author(s) 2022

\begin{abstract}
Emotional and behavioral problems (EBPs) frequently occur in young autistic children. Discrepancies between parents and other informants are common but can lead to uncertainty in formulation, diagnosis and care planning. This study aimed to explore child and informant characteristics are associated with reported child EBPs across settings. Participants were 83 4-8-year-old autistic children and their parents and teachers in the Autism Spectrum Treatment and Resilience (ASTAR) study. Questionnaires of child EBPs were completed by parents and teachers, and self-reported parenting stress and wellbeing measures were obtained. An observation of parent-child/researcher-child interaction was also completed. Parents reported more EBPs than teachers and parent-teacher agreement was low, particularly for emotional problems. Greater parenting stress and being verbal was associated with more parent- but not teacher-reported EBPs. More observed behaviors that challenge were displayed by minimally verbal children. More parenting stress could be associated with the presence of more EBPs in the home; alternatively, parenting stress may confound reports. It is essential for assessments of EBPs in autistic children to take a multi-informant approach. Better understanding of the associations between informant characteristics and informant discrepancies of EBPs is needed.
\end{abstract}

Keywords Autism $\cdot$ Child emotional and behavioral problems $\cdot$ Informants $\cdot$ Agreement $\cdot$ Discrepancies

\section{Introduction}

Melanie Palmer and Joanne Tarver are joint first authors and Tony Charman and Emily Simonoff are joint senior authors.

Melanie Palmer

melanie.palmer@kcl.ac.uk

1 Department of Child and Adolescent Psychiatry, King's College London, Institute of Psychiatry, Psychology \& Neuroscience, London, UK

2 Department of Psychology, School of Life and Health Sciences, Aston University, Birmingham, UK

3 Department of Biostatistics and Health Informatics, King's College London, Institute of Psychiatry, Psychology \& Neuroscience, London, UK

4 Newcomen Neurodevelopmental Centre, Evelina Children's Hospital, Guy's and St Thomas NHS Foundation Trust, London, UK

5 Service for Complex Autism \& Associated Neurodevelopmental Disorders, South London and Maudsley NHS Foundation Trust, London, UK

6 Department of Psychology, King's College London, Institute of Psychiatry, Psychology \& Neuroscience, London, UK
Autism Spectrum Disorder is a neurodevelopmental condition associated with difficulties in social communication, and the presence of restricted and repetitive interests and sensory processing difficulties (American Psychiatric Association, 2013). Emotional and behavioral problems (EBPs) are common in autistic $^{1}$ individuals (Lai et al., 2019). As many as $70 \%$ of autistic children may meet criteria for a co-occurring psychiatric condition; attention deficit hyperactivity disorder (ADHD), anxiety disorders and oppositional defiant disorder (ODD) are the most prevalent (Salazar et al., 2015; Simonoff et al., 2008). EBPs can also be associated with behaviors that challenge (BTC). ${ }^{2}$

\footnotetext{
1 We use 'autism' to refer to Autism Spectrum Disorder (ASD; DSM-5) and 'autistic children' as this is the preferred term of some of the autism community (Kenny et al., 2016).

${ }^{2}$ BTC are typically defined as a behavior that puts the individual, those involved in their care or services at risk of harm or exclusion, due to their intensity, frequency or duration (National Institute for Health and Care Excellence, 2015; Royal College of Psychiatrists, British Psychological Society, \& Royal College of Speech and Language Therapists, 2007). This term was chosen as it was preferred by our IAMHealth PPI panels.
} 
For example, anxiety is associated with oppositional behavior, hyperactivity, aggression and meltdowns (Sukhodolsky et al., 2020; Tarver et al., 2021a). For autistic individuals EBPs persist over time (Simonoff et al., 2013; Stringer et al., 2020), impact on quality of life (Mason et al., 2018) and are associated with more parental distress (Yorke et al., 2018).

\section{Informant Discrepancies in the Assessment of EBPs}

To conduct a thorough assessment of EBPs in childhood, guidelines suggest assessment of impairment across settings (National Institute for Health \& Care Excellence, 2013). The use of multiple informants (e.g. parent and teacher) and naturalistic observation ensures a comprehensive understanding of the individual across contexts (Mash \& Hunsley, 2005). Informant discrepancies, where informants provide different reports of similar constructs, are expected and commonplace during assessments of EBPs in autism (Stratis \& Lecavalier, 2015). This can lead to uncertainty in clinical decision making and treatment planning, but may give information on factors driving or maintaining EBPs.

There are a range of factors, not related to measurement error, that may influence reporting of child EBPs and lead to informant discrepancies. A key factor is situational specificity (De Los Reyes et al., 2013); the nature of the settings in which different individuals interact with the child could impact how informants understand and report EBPs. For example, the structured nature of the school environment may be associated with different displays of EBPs at school. Discrepancies across reports may therefore yield important information on how the child behaves in different contexts and can be used to inform environmental modification and intervention for EBPs.

Levels of EBPs may vary across settings and literature comparing reports from parents and teachers of EBPs among autistic children are mixed. Some studies report higher levels of teacher-reported oppositionality and anxiety (Reed \& Osborne, 2013), whereas others report higher levels of parent-reported EBPs compared to teachers (Jepsen et al., 2012; Llanes et al., 2020). Although comparable to agreement in the general population (Achenbach et al., 1987), levels of agreement between parents and teachers on reports of EBPs for autistic children are often moderate at best (Stratis \& Lecavalier, 2015). Whilst it is likely that situational specificity is playing a role, parentteacher agreement in autism remains low when teachers and parents are observing and reporting on child behavior in the home (Reed \& Osborne, 2013). This indicates that factors other than situational specificity are likely to influence reports of EBPs and better understanding of these factors is warranted. Furthermore, few studies have explored how observational measures of child behavior, often considered the 'gold standard', are associated with other informant reports of EBPs among autistic children.

\section{Child Characteristics Associated with Informant Reports}

\section{IQ and Verbal Language Level}

Verbal language level varies greatly in autism, and IQ, which shows strong overlap with verbal language level (Bal et al., 2016), is associated with reports of EBPs in autism. Parents of minimally verbal autistic children report higher levels of child irritability and hyperactivity than parents of verbally fluent children (Fok \& Bal, 2019). However, inconsistent relationships between informant reports of levels of anxiety and IQ have been found (see Kent \& Simonoff, 2017; Mingins et al., 2020 for reviews); some studies report greater parent-reported anxiety in autistic individuals with higher IQ (Chandler et al., 2016; Hallett et al., 2013; Salazar et al., 2015) and others do not find this association (Simonoff et al., 2008). Assessment of emotional problems is likely to be more difficult in minimally verbal individuals due to limited ability to communicate internal states. However, when considering the effects of IQ on informant agreement, evidence suggests that as IQ increases, informant agreement about autistic children's emotional problems decreases (Stratis \& Lecavalier, 2015). It was suggested that individuals with lower IQ show less varied behavior across contexts, or that caregiver discussions about EBPs may be more frequent in children and young people with low IQ leading to greater agreement.

\section{Autism Severity}

There are also mixed findings as to whether autism severity is associated with levels of reported EBPs. Some evidence suggests autism severity is associated with increased risk of meeting diagnostic criteria for agoraphobia and night terrors on a parent-informed psychiatric interview, but not other common co-occurring conditions including social anxiety and disruptive behavior disorders (Salazar et al., 2015). In another population-based cohort of autistic children, autism severity was not associated with increased risk for co-occurring conditions (Simonoff et al., 2012). Furthermore, Stratis and Lecavalier (2017) found no evidence that autism severity was associated with parentteacher agreement of child EBPs; however, Levinson et al. (2021) found that autism severity predicted disagreement between parent and teacher reports of EBPs. 


\section{Severity of EBPs}

Informant discrepancies could be a marker of the severity of child EBPs. Discrepancies may be more likely if child EBPs are less severe and/or frequent leading to differing interpretations between informants (De Los Reyes et al., 2009). However, in non-autistic children, there is evidence that occurrences of observed behavior specific to one context and interaction partner (e.g. parent or examiner) are related to higher levels of parent- or teacher-reported disruptive behavior (De Los Reyes et al., 2009). To the best of our knowledge, relationships between observations of behavior in a third context and parent and teacher reports of EBPs has not been explored in autism. This provides an important area for autism research given that children less able to modulate behavior across multiple contexts may be more likely to show more stable and severe EBPs (Granic \& Patterson, 2006; Jones et al., 1975).

\section{Informant Characteristics Associated with Informant Reports}

\section{Parenting Stress and Wellbeing}

Reports of EBPs are also likely to be influenced by a host of characteristics related to the informant, such as stress and wellbeing. Parents of autistic children report higher levels of stress than parents of non-autistic children and other developmental disabilities (Estes et al., 2013) and there is an established bidirectional relationship between parental distress and child EBPs in autism (Yorke et al., 2018). Parenting stress and wellbeing might be associated with reports of EBPs in several ways, leading to disagreement between different informants. First, parenting stress may reduce parental capacity to cope, perhaps biasing parental views of child behavior (Najman et al., 2000). Second, children of distressed parents may show elevated levels of problem behavior that is restricted to the home environment or during interactions with parents. Third, the relationship between parental distress and parent-reported child EBPs may be due to shared method variance (Podsakoff et al., 2003). There is some evidence to show that levels of parenting stress are associated with parental reports of certain domains of child functioning, such as behavioral problems, but not linked with clinician ratings of child functioning (Schwartzman et al., 2021). Exploration of relationships between parenting stress and reports of EBPs in other settings (such as teacher-reported EBPs and observational measures of child behavior) is limited. If parenting stress is associated with reports of EBPs by other informants, this may suggest that stress does not play a role in confounding parental reports of EBPs, or that parenting stress is elevated because EBPs are primarily displayed at home.
Teacher Characteristics Associated with Informant Reports

Various factors can also influence teacher reports of child EBPs among autistic children and could result in different reports of EBPs when compared to parents. Teacher reports are likely to be influenced by the structure and expectations of classroom activities and situations in schools. Further, teachers observe children in situations that parents may be less likely to see, including interactions with peers and performance in directed tasks. Another factor may be the type of school a teacher is employed in, and research has found that teachers working with autistic children in mainstream settings report higher levels of general, but not social anxiety, compared to teachers in specialist education settings (Adams et al., 2018). It may be that EBPs may be more commonly observed, and therefore less salient to teachers in specialist educational settings; saliency is a factor found to impact reported EBPs (Karver, 2006). Differences could also reflect a commonly reported, albeit inconsistent, finding of a relationship between higher IQ and greater anxiety in autism (e.g. Chandler et al., 2016), or true variations in anxiety displayed across contexts.

\section{Aims of the Current Study}

Given the varied findings in the literature of factors associated with reports of EBPs in autistic children, there were three key objectives of this study. First, we compared levels of EBPs in autistic children reported by parents and teachers. We hypothesized that parents would report higher levels of EBPs than teachers. Second, we explored whether child and informant characteristics were associated with levels of parent-, teacher- and researcher-rated observed EBPs. We hypothesized that less verbal language, greater autism severity and higher rates of researcher-rated observed behaviors that challenge (BTC; e.g. non-compliance, hyperactivity) would be associated with more parent- and teacherreported EBPs, and that autism severity and less verbal language would be associated with more researcher-rated observed BTC. We expected that higher self-reported parenting stress and lower parental wellbeing would correlate with greater parent-reported EBPs, and that special school placement would be associated with lower teacher-reported EBPs as teachers in these settings are likely to have differing thresholds and have more support for child EBPs. We did not have a specific hypothesis for whether parent characteristics would be associated with teacher-reported and researcher-rated observed child EBPs, but instead explored the associations between parental reports of parenting stress and wellbeing and these measures. Finally, we explored agreement between parent and teacher reports of EBPs and whether informant agreement between teachers and parents varied according to score severity. We hypothesized that 
parent-teacher agreement would be modest, but that agreement would increase as the severity of parent and teacher rated EBPs increased.

\section{Method}

\section{Participants}

Participants were 83 young autistic children and their parents (see Table 1 for further details) participating in the Autism Spectrum Treatment and Resilience (ASTAR) study (Charman et al., 2021; Palmer et al., 2019), as part of the Improving Autism Mental Health (IAMHealth) research program (https://iamhealthkcl.net). Most children were male $(n=71$, $85.5 \%)$ and the mean age was 6.70 years $(S D=1.21$ years). Verbal language levels varied across the sample; 39 (47.0\%) were minimally verbal and $44(53.0 \%)$ were verbal (see description of verbal ability grouping in measures section below). In addition, 76 education professionals (mainly Class Teachers, but occasionally a school Special Educational Needs Coordinator, Learning Support Assistant, or Head Teacher; referred to hereafter as 'teachers' for simplicity) completed questionnaires about the children.

\section{Procedure}

Participants were enrolled in the ASTAR study, a non-randomized feasibility study followed by a pilot randomized controlled trial (RCT) of two novel parent-mediated group interventions. Ethical approval for the study was granted from NHS Camden and Kings Cross Research Ethics Committee (ref: 16/LO/1769). Written informed consent was obtained from all participating parents and teachers and child assent was obtained wherever appropriate.

Twenty-one parent-child dyads participated in the feasibility study and 62 in the pilot RCT, recruited from local autism diagnostic teams, education professionals, support groups, consented databases, and self-referral routes in four boroughs of South London. To be eligible to take part, the child had to have a clinical diagnosis of an Autism Spectrum Disorder and be between 4 years 0 months and 8 years 11 months at randomization. The study was designed to be as inclusive as possible, so no exclusion based on levels of child EBPs was used; nor for verbal language level or IQ. Additional inclusion/exclusion criteria can be found in Palmer et al. (2019).

Data collected during the baseline assessments were used in the current study. Parents completed questionnaires on their child's EBPs and their own parenting stress and wellbeing. With parental consent, the child's teacher completed questionnaires about the child's EBPs at school. The baseline assessment also involved a research visit where observations of parent-child/researcher-child interaction and autism severity were obtained.

\section{Measures}

\section{Sample Characterization}

Demographic information about the family was obtained using a bespoke questionnaire. Autism severity was measured using the Autism Diagnostic Observation Schedule - 2nd edition (ADOS-2, Lord et al., 2012). Verbal language grouping was based on the ADOS-2 module completed with the child ( minimally verbal $=$ Module 1 vs. verbal $=$ Module 2 or 3 ). To measure adaptive skills and functioning, the Adaptive Behavior Assessment System $-3^{\text {rd }}$ edition (ABAS-3, Harrison \& Oakland, 2015) was completed by parents. Scores on the ADOS-2 and the ABAS-3 for the sample are presented in Table 1.

\section{Parent-Reported Child Emotional and Behavioral Problems}

Parent-reported child irritability and hyperactivity was measured using the Irritability and Hyperactivity subscales of the Aberrant Behavior Checklist (ABC, Aman \& Singh, 1994). The ABC is an informant report measure of EBPs developed for use in populations with developmental disabilities and is widely used in autism intervention studies (e.g. Bearss et al., 2015). Items were rated on a 4-point scale ranging from 'not at all a problem' to 'the problem is severe in degree' and summed to produce total subscale scores, with higher totals signifying more irritability and hyperactivity. The $\mathrm{ABC}$ has established reliability and validity (Aman \& Singh, 1994).

Parent-reported child EBPs were also measured using the Emotional (19 items; e.g. spends a lot of the day feeling worried) and Behavioral (15 items; e.g. too much energy) Problems subscales of the Assessment of Concerning Behaviour (ACB). The ACB is a new measure developed specifically for use in autistic children and young people (Tarver et al., 2021b). Items were rated on a 5-point sliding scale anchored by opposing responses ('not at all' to 'very much') and summed to produce total subscale scores. Higher scores indicate more EBPs. Good internal validity for parent-reported ACB Emotional $(\alpha=0.87)$ and Behavioral Problems $(\alpha=0.86)$ subscales was found for this sample.

\section{Teacher-Reported Child Emotional and Behavioral Problems}

Teachers completed the ABC Irritability and Hyperactivity subscales (Aman \& Singh, 1994) and the teacher version of the ACB (Tarver et al., 2021b). In this sample, internal 
Table 1 Characteristics of the sample $(N=83)$

\begin{tabular}{|c|c|c|c|}
\hline \multicolumn{2}{|l|}{ Demographic characteristics } & $n$ & $\%$ \\
\hline \multicolumn{4}{|l|}{ Child gender } \\
\hline \multicolumn{2}{|l|}{ Male } & 71 & $85.5 \%$ \\
\hline \multicolumn{4}{|l|}{ Child ethnicity $^{\mathrm{a}}$} \\
\hline \multicolumn{2}{|l|}{ White } & 44 & $53.7 \%$ \\
\hline \multicolumn{2}{|l|}{ Asian/Asian British } & 8 & $9.8 \%$ \\
\hline \multicolumn{2}{|l|}{ Black/Asian British } & 14 & $17.1 \%$ \\
\hline \multicolumn{2}{|l|}{ Mixed/Multiple ethnicities } & 15 & $18.3 \%$ \\
\hline \multicolumn{4}{|l|}{ School placement ${ }^{\mathrm{b}}$} \\
\hline \multicolumn{2}{|l|}{ Specialist school } & 32 & $39.0 \%$ \\
\hline \multicolumn{2}{|l|}{ Mainstream school } & 37 & $45.1 \%$ \\
\hline \multicolumn{2}{|l|}{ Specialist unit in mainstream school } & 13 & $15.9 \%$ \\
\hline \multicolumn{4}{|l|}{ Parent informant ${ }^{\mathrm{c}}$} \\
\hline \multicolumn{2}{|l|}{ Mother } & 76 & $91.6 \%$ \\
\hline \multicolumn{2}{|l|}{ Father } & 5 & $6.0 \%$ \\
\hline \multicolumn{2}{|l|}{ Grandmother } & 2 & $2.4 \%$ \\
\hline \multicolumn{4}{|l|}{ Parental education level $^{\mathrm{c}}$} \\
\hline \multicolumn{2}{|l|}{ No formal qualifications } & 10 & $12.2 \%$ \\
\hline \multicolumn{2}{|l|}{ General Certificate of Secondary Education or equivalent } & 9 & $11.0 \%$ \\
\hline \multicolumn{2}{|c|}{ General Certificate of Education Advanced Level (A levels) or equivalent } & 10 & $12.2 \%$ \\
\hline \multicolumn{2}{|c|}{ Vocational qualifications (NVQ, City and Guilds or equivalent) } & 13 & $15.9 \%$ \\
\hline \multicolumn{2}{|l|}{ Undergraduate tertiary degree } & 16 & $19.5 \%$ \\
\hline \multicolumn{2}{|l|}{ Postgraduate tertiary degree } & 24 & $29.3 \%$ \\
\hline \multicolumn{4}{|l|}{ Parental employment status ${ }^{\mathrm{b}, \mathrm{c}}$} \\
\hline \multicolumn{2}{|l|}{ Not in paid employment } & 40 & $48.8 \%$ \\
\hline \multicolumn{2}{|l|}{ In part-time paid employment } & 25 & $30.5 \%$ \\
\hline \multicolumn{2}{|l|}{ In full-time paid employment } & 17 & $20.7 \%$ \\
\hline \multicolumn{4}{|l|}{ Annual household income ${ }^{\mathrm{d}}$} \\
\hline Less than $£ 20,000$ & & 23 & $35.9 \%$ \\
\hline$£ 20,000-£ 39,999$ & & 14 & $21.9 \%$ \\
\hline$£ 40,000-£ 59,999$ & & 8 & $12.5 \%$ \\
\hline$£ 60,000-£ 79,999$ & & 10 & $15.6 \%$ \\
\hline Greater than $£ 80,000$ & & 9 & $14.1 \%$ \\
\hline Other clinical characteristics & $M$ & $S D$ & Observed range \\
\hline $\begin{array}{l}\text { Observed child behaviors that challenge rate/ per minute } \\
\text { (OSCA-ABP) }\end{array}$ & 2.00 & 1.81 & $0.05-8.74$ \\
\hline Autism severity (ADOS-2 CSS) & 7.53 & 1.90 & $1-10$ \\
\hline Adaptive behavior (ABAS-3) & 62.51 & 12.79 & $45-100$ \\
\hline Parenting stress (APSI) ${ }^{\mathrm{b}}$ & 22.46 & 9.58 & $5-49$ \\
\hline Parental wellbeing (SWEMWBS) ${ }^{\mathrm{b}}$ & 21.08 & 4.20 & $7-30.70$ \\
\hline
\end{tabular}

$N=83$, valid \% reported

ABAS-3 Adaptive Behavior Assessment System - 3rd edition, ADOS-2 CSS Autism Diagnostic Observation Schedule, second edition, Calibrated Severity Score (0-10), APSI Autism Parenting Stress Index (0-65), OSCA-ABP Observation Schedule for Children with Autism-Anxiety, Behaviour and Parenting (0+), SWEMWBS Short Warwick-Edinburgh Mental Well-being Scale (7-35)

${ }^{a} n=81$ Data was missing for one child and one did not wish to answer. White=English/Welsh/Scottish/Northern Irish/Irish/British/Other White ethnicity, Black/Black British=African/Caribbean/Other Black ethnicity, Asian/Asian British=Indian/Pakistani/Bangladeshi/Chinese/Other Asian ethnicity, Mixed/Multiple ethnicities = White and Black Caribbean/White and Black African/White and Asian/Other Mixed ethnicity

${ }^{\mathrm{b}} n=82$. Data was missing for one child

${ }^{\mathrm{c}}$ Refers to parent involved in completing the questionnaires/observation

${ }^{\mathrm{d}} n=64$. Data was missing for one child and 18 did not wish to answer 
validity of teacher ACB Emotional and Behavioral Problems was $\alpha=0.77$ and $\alpha=0.88$ respectively.

\section{Observed Behaviors that Challenge}

The rate of child behaviors that challenge (BTC) observed during an 18 to 22-min structured parent-child/ researcher-child interaction, the Observation Schedule for Children with Autism Spectrum Disorders - Anxiety, Behaviour and Parenting (OSCA-ABP, Palmer, Paris Perez et al., 2020), were coded from video-recordings by researchers. During the observation, six parent-led (shared task, shared game, separation, reunification and tidy up, homework sheet and walking along a line) and two researcherled (mystery box and an unopenable snack jar with reward delay) tasks were completed that aimed to elicit observable BTC by tapping into uncertainty and novelty, transition, turn taking, sensory processing, compliance, frustration and reward delay, see Palmer, Paris Perez et al. (2020) for further details. The specific materials used in each task were differentiated by child verbal ability group based on the ADOS-2 assessment modules, to take into account differences in expressive language and age, but were designed to be analogous in function. The frequencies of a range of child behaviors (destructive behavior, aggression towards themselves and others, frustrated vocalizations, non-compliance, avoidance and reassurance seeking) observed during the OSCA-ABP were coded and summed to produce the total child BTC score. As the duration of the measure varied, the rate of child BTC per minute was calculated by dividing the total BTC count by the duration. The OSCA-ABP child BTC rate has demonstrated good inter-rater reliability amongst the current sample of verbal $(I C C=0.92,95 \%$ $C I=0.88,0.96)$ and minimally verbal children $(I C C=0.77$, 95\% $C I=0.66,0.89$ ) (Palmer, Paris Perez et al., 2020), and provides a global measure of EBPs, as it covers both anxious and disruptive behavior.

\section{Parenting Stress}

Parenting stress associated with core and co-occurring symptoms of autism was measured using the Autism Parenting Stress Index (APSI, Silva \& Schalock, 2012). Thirteen items were rated on a 5-point scale ('not stressful' to 'so stressful we feel we can't cope'), summed to produce a total score with higher scores indicating more parenting stress. The APSI has acceptable internal validity and test-retest reliability (Silva \& Schalock, 2012).

\section{Parental Wellbeing}

Parents reported on their own wellbeing using the Short Warwick Edinburgh Mental Well-Being Scale (SWEMWBS,
NHS Health Scotland, University of Warwick and University of Edinburgh, 2008). The SWEMWBS consists of seven positively worded items that tap into wellbeing, summed to produce a total score with higher scores indicating higher wellbeing. The SWEMWBS construct and convergent validity of the measure has been established (Ng Fat et al., 2017).

\section{Data Analysis}

Data analysis was conducted in Stata 15 (StataCorp, 2015). All variables were assessed for normality. Teacher ABC child irritability scores violated the assumptions of normality and where possible, the appropriate non-parametric tests were used. Where there was no non-parametric alternative, we conducted sensitivity analysis using the log transformation of the variable. The interpretation of the results was unchanged when using the log-transformed variable, so the untransformed variable was retained and used for all parametric analyses. First, we examined differences in mean parent- and teacher-reported scores on the $\mathrm{ABC}$ and $\mathrm{ACB}$ subscales using paired sample $t$-tests and Cohen's $d$ was used to calculate the size of the effects between the groups.

Next, we used a hypothesis-driven approach to test whether child and informant characteristics were associated with levels of parent-, teacher- and researcher-rated observed EBPs. First, we explored the correlations between all potential factors (i.e. child and informant characteristics) and measures of EBPs. To dichotomize school placement, specialist units in a mainstream school were included with mainstream schools, and specialist only schools were separate. This is consistent with previous research that has combined special units in mainstream schools in this way (e.g. Simonoff et al., 2019).

We then conducted a series of multivariate multiple linear regression models using the sem command to explore the strength of association between each factor and both parent and teacher-reported EBPs whilst controlling for the other characteristics. As parent- and teacher-reports were obtained using the same measure, they could be entered into the same model (one for each subscale), which increased efficiency and reduced type 1 errors from multiple testing. Where significant associations were found between an independent and dependent variable (either parent- or teacher-reported scores), post-estimation tests were used to compare the coefficients (i.e. testing if the strength of the association between an independent variable and parent-reported scores was significantly different from the association between that same variable and teacher-reported scores).

As observed child BTC were rated on a different scale to the measures completed by parents and teachers, associations with observed BTC were tested using a univariate multiple linear regression model. Independent variables included child verbal language, parenting stress and parental 
Table 2 Differences in mean scores for parent- and teacher-reported child emotional and behavioral problems and inter-rater agreement across measures

\begin{tabular}{|c|c|c|c|c|c|c|c|c|c|c|c|c|}
\hline \multirow[t]{4}{*}{ Measure } & \multicolumn{3}{|c|}{ Descriptives } & \multicolumn{9}{|c|}{ Differences in parent-teacher means and inter-rater agreement } \\
\hline & \multicolumn{3}{|c|}{$\begin{array}{l}\text { Parent-reported child emotional } \\
\text { and behavioral problems }{ }^{\mathrm{a}}\end{array}$} & \multicolumn{3}{|c|}{$\begin{array}{l}\text { Teacher-reported child emo- } \\
\text { tional and behavioral problems }\end{array}$} & & & & & & \\
\hline & \multirow[t]{2}{*}{$M$} & \multirow[t]{2}{*}{$S D$} & \multirow[t]{2}{*}{ Observed range } & \multirow[t]{2}{*}{$M$} & \multirow[t]{2}{*}{$S D$} & \multirow[t]{2}{*}{ Observed range } & \multicolumn{3}{|c|}{$\begin{array}{l}\text { Tests comparing mean } \\
\text { parent and teacher } \\
\text { reports }^{\mathrm{b}}\end{array}$} & \multicolumn{3}{|c|}{$\begin{array}{l}\text { Intraclass correlation } \\
\text { coefficients }(I C C)^{\mathrm{b}}\end{array}$} \\
\hline & & & & & & & $t$ or $W$ & $p$ & $d$ & $I C C$ & $95 \% C I$ & $p$ \\
\hline Irritability (ABC) & 16.37 & 10.30 & $0-44$ & 9.16 & 10.08 & $0-44$ & $4.09^{c}$ & $<.001$ & 0.50 & .16 & $-.04, .36$ & .047 \\
\hline Hyperactivity (ABC) & 24.09 & 13.34 & $1-48$ & 16.93 & 12.51 & $0-47$ & 3.89 & $<.001$ & 0.45 & .30 & $.08, .49$ & .001 \\
\hline Behavioral problems (ACB) & 22.65 & 11.43 & $2-49$ & 16.68 & 11.21 & $0-56$ & 3.67 & $<.001$ & 0.42 & .27 & $.06, .47$ & .003 \\
\hline Emotional problems (ACB) & 21.48 & 13.09 & $0-59.11$ & 14.66 & 8.92 & $1-42$ & 3.76 & $<.001$ & 0.43 & .04 & $-.15, .24$ & .350 \\
\hline
\end{tabular}

$N=83 . \mathrm{ABC}=$ Aberrant Behavior Checklist (Irritably 0-45; Hyperactivity 0-48); $\mathrm{ACB}=$ Assessment of Concerning Behaviour (Externalizing $0-60$; Internalizing 0.76)

${ }^{\mathrm{a}} n=82$. Data was missing for one child

${ }^{\mathrm{b}} n=76$. Teacher data was missing for seven children

${ }^{\mathrm{c}}$ Non-parametric alternative to paired $t$-test (Wilcoxon signed-rank test) and Pearson's correlation (Spearman's rho) used due to the teacher ABC irritability variable being positively skewed. Possible range of scores for each measure are in brackets below

wellbeing. School placement was not included in this regression given the overlap between verbal language grouping and school placement and we did not have a clear hypothesis about how school placement would influence researcherrated observed BTC above and beyond verbal language.

Inter-rater agreement between parents and teachers was explored in two ways. First, we obtained the intraclass correlation coefficients (ICCs) and 95\% confidence intervals (CIs) to assess agreement across parent and teacher scores on the $\mathrm{ABC}$ and the ACB. $F$ tests were used to test whether the ICC were significantly different from zero. Bland-Altman plots (BA, Bland \& Altman, 1995) were also produced to visually depict systematic trends in informant agreement across the range of scores on the ABC and ACB subscales. We tested whether parent and teacher agreement was associated with score severity on each subscale using Spearman's rho (as in Bland \& Altman, 1995).

\section{Results}

\section{Group Differences Between Parent- and Teacher-Reported Emotional and Behavioral Problems}

Table 2 displays the descriptive statistics and the results of the $t$-tests comparing parent and teacher scores on the ABC and ACB subscales and associated effect sizes. As hypothesized, parents reported significantly more irritability and hyperactivity on the ABC and more emotional and behavioral problems on the ACB compared to teachers; all effect sizes were moderate.

\section{Factors Associated with Parent- and Teacher-Reported Emotional and Behavioral Problems}

Supplementary Table S1 displays the bivariate correlations between the child and informant characteristics and parent-, teacher- and researcher-reports of child EBPs (see Table 1 for descriptives of the clinical measures). Parenting stress and wellbeing were significantly positively correlated with the majority of parent-reported EBPs ( $r$ 's ranged from $0.37-0.61, p$ 's all $<0.001$ and $r$ 's were $-0.20--0.51$, $p$ 's from 0.071 to $<0.001$ for parenting stress and wellbeing, respectively). Observed BTC was positively correlated with teacher-reported behavioral problems on the ACB $(r=0.23$, $p=0.048$ ).

Table 3 displays the standardized coefficients and their associated $p$ values for the multivariate linear multiple regression models examining parent- and teacher-reported EBPs. Figure 1 is a forest plot showing the standardized coefficients for these models.

Higher parental ABC irritability scores were uniquely associated with the child being verbal and greater parenting stress. Post-estimation tests showed that children whose parents reported greater parenting stress had significantly higher parent-, but not teacher-reported, child irritability $(p<0.001)$. The post-estimation test for verbal language grouping was not significant $(p=0.093)$. 


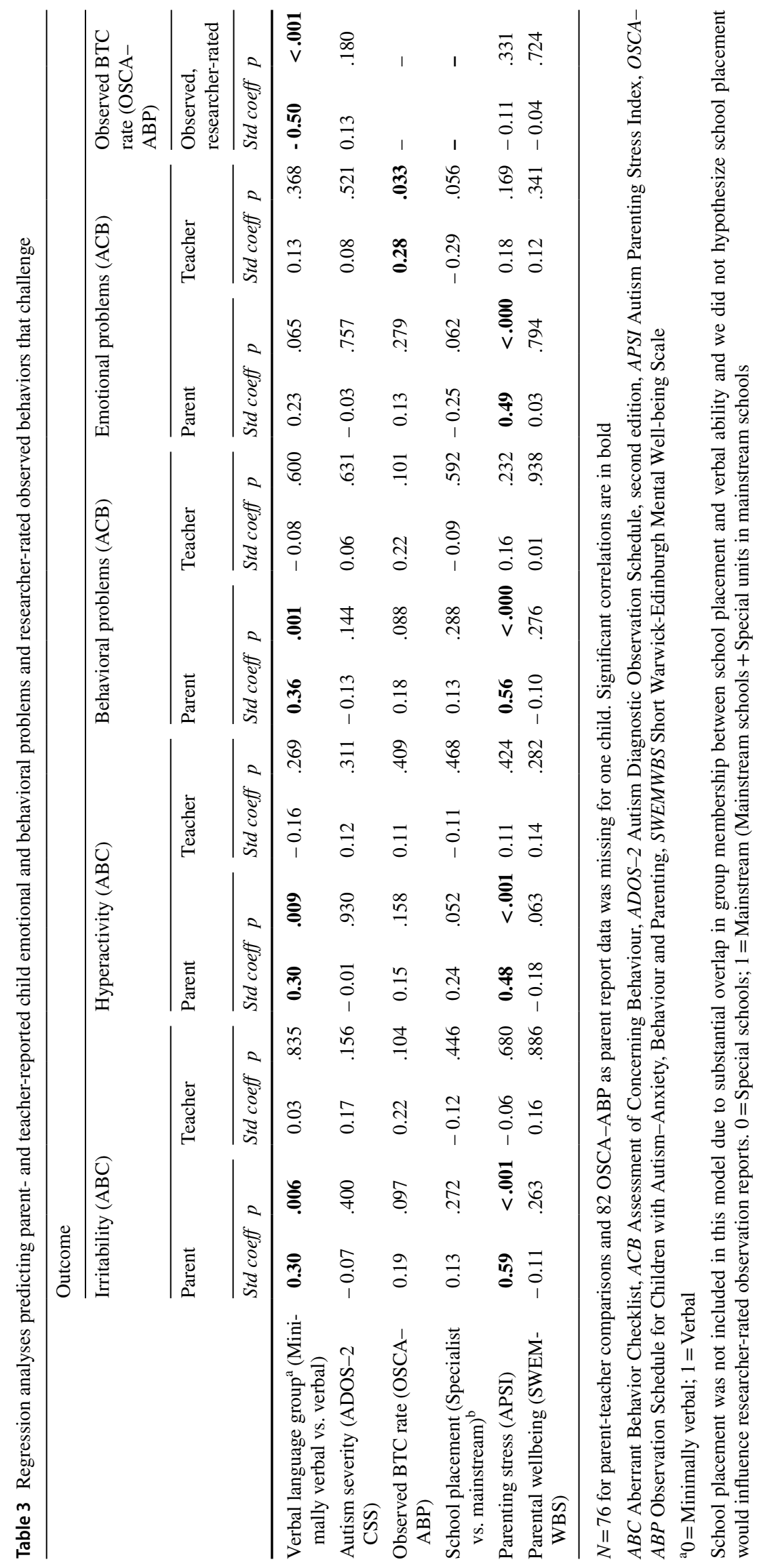



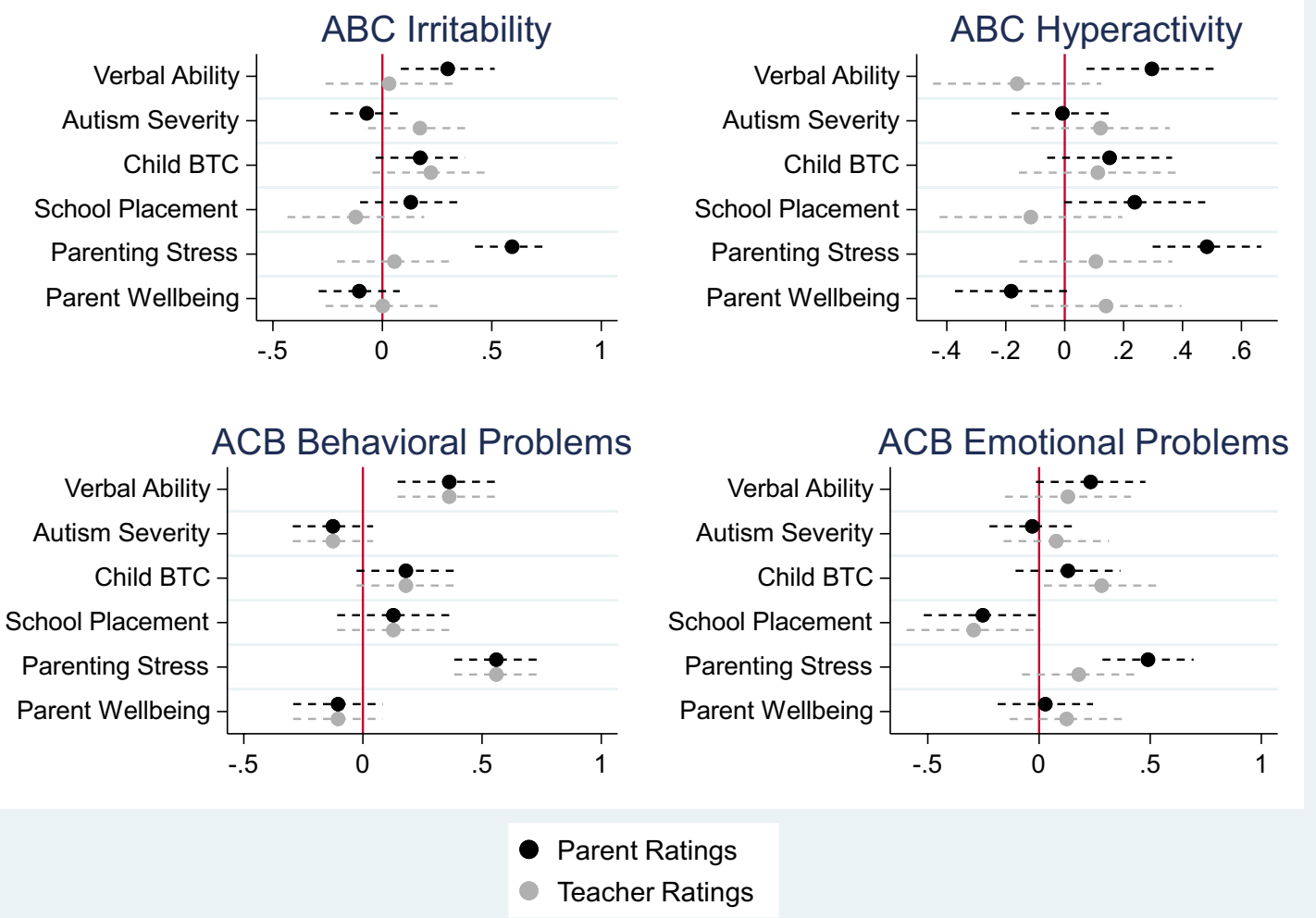

Fig. 1 Forest plots showing the standardized coefficients for the multivariate regression models predicting parent- and teacher-reported EBPs

For parent-reported $\mathrm{ABC}$ hyperactivity, the child being verbal and greater parenting stress were associated with higher hyperactivity scores. Post-estimation tests showed that these factors were significantly associated with parent ( $p=0.001$ and 0.002 respectively) but not teacher reports of hyperactivity.

For ACB behavioral problems, children who were verbal and had parents reporting greater parenting stress had significantly higher parent-reported behavioral problems; post-estimation tests showed these variables were significant correlates of parent-reported behavioral problems when compared to teacher reports $(p=0.004$ and $p=0.004$ ).

For the ACB emotional problems scores, higher parenting stress was associated with more child emotional problems, with post-estimation tests showing that higher levels of parenting stress were associated with higher levels of parent-reported, but not teacher-reported, child emotional problems $(p=0.013)$. For teachers, more observed BTC was significantly associated with higher teacher reports of child emotional problems, however, post-estimation tests showed that when compared to parent-report, observed child BTC was not significantly associated with teacher-reported emotional problems $(p=0.703)$.

\section{Factors Associated with Observed Child Behaviors that Challenge}

Supplementary Table S1 displays the correlations between the child and informant characteristics and researcher-rated observed child BTC rates. Being minimally verbal was associated with more observable child BTC. The standardized coefficients and associated $p$ values for the univariate multiple linear regression model examining researcher-rated observed child BTC rates are also displayed in Table 3. When controlling for other variables, being minimally verbal was the only factor associated with more observable child BTC.

\section{Agreement between Parent- and Teacher-Reported Emotional and Behavioral Problems}

Table 2 presents the ICCs between parent and teacher scores and associated $p$ values on the ABC and ACB measures. The ICCs examining agreement between parents and teachers ranged from 0.04 (child emotional problems) to 0.30 (child hyperactivity). ICCs were statistically significant for all variables with the exception of ACB child emotional problems. 
Informant agreement and variation in agreement by score severity was further examined using Bland-Altman plots and the accompanying regression analysis (see Supplementary Fig. S1). Supplementary Table S2 presents the mean differences in parent and teacher scores and combined parent-teacher scores. No significant relationships between the difference scores and the average combined scores were found for the $\mathrm{ABC}$ irritability, $\mathrm{ABC}$ hyperactivity and $\mathrm{ACB}$ behavioral problems (see Supplementary Fig. S1 for $\rho$ and $p$ values). However, for the ACB emotional problems, there was a significant relationship indicating that agreement varied based on severity of emotional problems $(\rho=0.29$, $p=0.011$ ), with a general pattern of larger discrepancy between informants as emotional problem scores increase.

\section{Discussion}

This study compared levels and investigated child and informant characteristics associated with child EBPs and reports from three different informants (parents, teachers and researchers). It also explored agreement between parent- and teacher-reported EBPs.

\section{Levels of Parent- and Teacher-Reported Emotional and Behavioral Problems}

As hypothesized, in the current sample of 4-8-year-old autistic children, parents reported more EBPs than teachers and this pattern held across both emotional and behavioral problems. This aligns with previous literature in non-autistic (Rescorla et al., 2014) and autistic populations (Chandler et al., 2016; Jepsen et al., 2012). The differences in levels of EBPs reported in home and educational settings lends support for the notion of situational specificity, although because the reports are provided by different individuals, other informant characteristics cannot be ruled out. The nature of these different environments is likely to influence which behaviors are displayed. One possibility is that the structured, routine-based school environment reduces the likelihood of a child encountering unexpected events or stimuli, a key trigger for EBPs in autism (Bearss et al., 2015). A further explanation, often described anecdotally by parents and clinicians, is a delayed display of the pressures faced by autistic children during a typical school day. It may be that autistic children are 'holding in' manifestations of EBPs (Bearss et al., 2015) in school environments resulting in reports of less EBPs by teachers and greater manifestations of EBPs when with parents. This may only be relevant for a sub-group of autistic individuals who have the cognitive ability to reflect and greater self-control to modify their behavior. Indeed, in this study, the child being verbal (suggesting higher IQ and more cognitive ability) was generally associated with higher parent-, but not teacher-, reported EBPs.

\section{Factors Associated with Parent- and Teacher-Reported Emotional and Behavioral Problems}

We also explored child and informant characteristics associated with parent-, teacher- and researcher-rated EBPs. For emotional problems, higher levels of parenting stress were associated with more parent-reported child emotional problems. For behavioral problems, higher levels of parenting stress and the child being verbal were associated with increased parent-reported behavioral problems. This finding is at odds with previous research that has reported more irritability and hyperactivity among minimally verbal children, albeit in a sample of older autistic children and young people aged 6-18 years (Fok \& Bal, 2019). This inconsistent finding could also be due to difficulties in measuring EBPs in autistic children, particularly among those who are minimally verbal or have an intellectual disability (Flynn et al., 2017). However, the relationship between the child being verbal and elevated parent-reported behavioral problems was consistent across the measures used in this study, including the ACB, a measure of EBPs developed specifically for autistic populations and shown to be reliable and valid across the spectrum of developmental ability in autism (Tarver et al., 2021b). Interestingly, parental wellbeing was not a correlate of EBPs. It may be that stress related to the parenting role has a stronger relationship with child EBPs than more general parent wellbeing (e.g. feeling relaxed and confident, can deal with day-to-day problems). This could be especially true given that the parenting stress measure used in this study was specific to stress related to autistic traits and co-occurring EBPs.

That parenting stress is associated with parent-reported EBPs but not teacher nor observed child difficulties in our study is important. This adds to previous research with both non-autistic and autistic children which found parenting stress is associated with greater discrepancies in reports of EBPs between parents and teachers (Langberg et al., 2010; van der Oord et al., 2006). The findings from this study could suggest that stress is influencing parent reports of EBPs in this sample (Najman et al., 2000); that the association between elevated parenting stress and parent-reported EBPs was consistent across multiple parent-reported measures acts as further support for this. However, it is also possible that parenting stress is elevated because EBPs are predominantly challenging in the home context, or in situations when children are more likely to be with their parents (e.g. in the supermarket, when on holiday). If so, the findings of this study may be suggestive of situational specificity, and that young autistic children are more likely to manifest EBPs 
with their parents. This is not to say that autistic children do not display EBPs at school, but instead, they become more visible when children get home (Bearss et al., 2015). When we tested whether the same characteristics were also associated with teacher reports of EBPs, a different pattern of results was found. None of the child or informant characteristics included in the models were associated with teacher reports of child irritability, hyperactivity, behavioral or emotional problems. Contrary to our hypothesis, verbal ability was not associated with teacher-reported EBPs. On the other hand, being minimally verbal was associated with more researcher-rated observed BTC, which is in line with other literature (e.g. Einfeld et al., 2011). No parental characteristics were associated with the rate of child BTC seen during this observation.

\section{Agreement Between Parent- and Teacher-Reported Emotional and Behavioral Problems}

In general, agreement between parent and teacher reports of EBPs was low. In line with non-autistic populations (e.g. Rescorla et al., 2014), agreement between parents and teachers appeared to be stronger for more observable behaviors (hyperactivity; behavioral problems), with lower agreement found for measures of irritability and emotional problems (domains which include questions interrogating internal states and mood). This pattern of findings is also similar to the levels of agreement reported between parents and teachers of young autistic individuals (Llanes et al., 2020; Stratis \& Lecavalier, 2015), although agreement between parents and teachers in the current sample is lower for emotional problems in particular. This may be in part due to the small sample size and lack of precision as confidence intervals were wide.

Agreement was also explored using Bland-Altman plots to visually depict trends in informant agreement. For the measures of child irritability, hyperactivity and behavior problems, there was no relationship between parent and teacher agreement and the severity of EBPs. However, for emotional problems, agreement varied based on how severe the scores were with a general pattern of larger differences between informants as emotional problems increased. This suggests that when emotional problems are reported as problematic in one context, they may not necessarily be problematic in another context. For example, anxiety could be more 'situation specific' than hyperactivity among autistic children. Another explanation may be that anxiety presents in atypical ways in autism (e.g. as repetitive or challenging behavior, Bearss et al., 2015; Kerns et al., 2014; Tarver et al., 2021a) and reliance on interpretation may explain lower agreement. However, it is of note that we did not find any association between parent-reported emotional problems and teacher-reported behavioral problems, and vice versa.
This suggests emotional problems rated by one informant are not interpreted as behavioral problems by the other.

\section{Clinical Implications}

Parent-teacher agreement on measures of EBPs is modest; young autistic children may show variations in EBPs across settings. A comprehensive assessment of EBPs should always take a cross-setting, multi-informant approach to assist with understanding triggers for EBPs, clinical decision-making and care planning. Young autistic children displaying elevated EBPs at home, may not display this at school, and vice versa. Another key clinical implication is that an assessment of parenting stress should be conducted during care planning for autistic children, as recommended by the National Institute for Health and Care Excellence guidance for assessment of autism and intellectual disability and challenging behavior (National Institute for Health \& Care Excellence, 2015, 2016). This is especially the case if discrepancies in parent and teacher reports, or observations of child EBPs are evident. An objective observation of the child in the home environment may aid clinician understanding of whether more parent-reported EBPs is because EBPs are primarily manifesting in the home environment. Given the differences in both the levels of EBPs and the modest agreement between parents and teachers, it may mean that different support for parents and teachers to help them manage child EBPs is required. It is important that discrepancies in informant reports are not dismissed as measurement error or informant bias. Clinicians should consider whether variations in informant report might reflect true situational variation in EBPs.

\section{Limitations and Future Research}

There are several limitations to the current study. There was limited information on the characteristics of teachers and schools, so it was not possible to explore if other characteristics (e.g. teaching experience, workplace stress, number of adults in the classroom) were associated with reports of EBPs. Future research could consider how teachers' confidence in supporting children with EBPs and their own levels of stress are associated with reports of EBPs. The observational measure was conducted in a third context and child BTC, whilst displayed primarily when interacting with their parent, was rated by a researcher. We did not conduct observations in either the home or school settings, so we are unable to tease apart the differences in contexts from the differences in informants. In addition, the study is correlational in nature so causality cannot be established. Future research using longitudinal designs should explore how reports of EBPs change over time and which factors 
are associated with discrepancies between informants both cross-sectionally and longitudinally. Finally, given that these data are drawn from a trial of a parent intervention for EBPs in autistic children it is possible the sample represents motivated families particularly concerned about EBPs, which may impact on generalizability.

\section{Conclusions}

The current study contributes to the literature on reported EBPs among young autistic children. It uses a well-defined, representative sample of young autistic children to explore whether various child and informant characteristics are associated with EBPs and discrepancies between informants. The study highlights that the use of multiple informants and naturalistic observation is important for the assessment of EBPs in young autistic children to obtain a comprehensive understanding of the individual across contexts and support clinical decision making (Mash \& Hunsley, 2005). Discrepancies between reports seem to be common and possible reasons for discrepancies should be explored; parenting stress and situational specificity may be particularly important. Assessment of EBPs could be supported by more objective measures, such as physiological response and independent observations of behavior, which could also be incorporated in evaluations of interventions designed to reduce EBPs in autistic children.

Supplementary Information The online version contains supplementary material available at https://doi.org/10.1007/s10803-021-05421-x.

Acknowledgements We are grateful to the families who were involved in the study and the IAMHealth parents' and autistic adults' panels who provide guidance and support throughout the trial. We would also like to acknowledge Elena Baker, Thomas Cawthorne, Lydia JohnsonFerguson, Moriya Maccabee and Sophie Webb for their assistance with data collection. We would also like to thank local professionals who assisted with identifying potential participants for the study, with particular thanks to Dr Fernando Salazar and the team at Croydon CAMHS, Dr Mark O'Leary, Sarran Bond and Jackie Sutherland and the team at Bromley Healthcare, Dr Shade Alu and Marion Drennan from the Croydon Child Development Centre, Dr Narad Muthura from the Mary Sheridan Children's Centre, Melissa Hendry and Maria Davies from Red Gates School and Marie Neave and Hannah Robinson from Drumbeat School.

Author Contributions ES, TC, SS, AP and VS obtained funding for the original study. MP and JT conceived the idea for the current manuscript, and MF explored a similar idea in her MSc thesis. Additionally, MP, JT and JPP were involved in recruiting participants and collecting the data. VCL assisted with the statistical analysis, interpretation of the results and drafting the manuscript. All authors read, made revisions and approved the final version.

Funding This manuscript summarizes independent research funded by the National Institute for Health Research (NIHR) under its Programme Grants for Applied Research programme (RP-PG-1211-20016). The views expressed in this manuscript are those of the authors and not necessarily those of the NHS, the NIHR or the Department of Health. $\mathrm{AP}$ and ES receives support from the NIHR through a Senior Investigator Award (NF-SI-0617-10120, NF-SI-0514-10073) and additionally receive support from the National Institute for Health Research (NIHR) Biomedical Research Centre at South London and Maudsley Foundation Trust (IS-BRC-1215-20018). VCL is supported by a Sir Henry Wellcome Postdoctoral Fellowship (213608/Z/18/Z).

\section{Declarations}

Conflict of interest TC has received consultancy fees from F. Hoffmann-La Roche Ltd. and Servier and royalties from Sage Publications and Guilford Publications. AP has received royalties from Western Psychological Services. All other authors declare no conflicts of interest.

Open Access This article is licensed under a Creative Commons Attribution 4.0 International License, which permits use, sharing, adaptation, distribution and reproduction in any medium or format, as long as you give appropriate credit to the original author(s) and the source, provide a link to the Creative Commons licence, and indicate if changes were made. The images or other third party material in this article are included in the article's Creative Commons licence, unless indicated otherwise in a credit line to the material. If material is not included in the article's Creative Commons licence and your intended use is not permitted by statutory regulation or exceeds the permitted use, you will need to obtain permission directly from the copyright holder. To view a copy of this licence, visit http://creativecommons.org/licenses/by/4.0/.

\section{References}

Achenbach, T. M., McConaughy, S. H., \& Howell, C. T. (1987). Child/ adolescent behavioral and emotional problems: Implications of cross-informant correlations for situational specificity. Psychological Bulletin, 101(2), 213-232.

Adams, D., Simpson, K., \& Keen, D. (2018). School-related anxiety symptomatology in a community sample of primary-school-aged children on the autism spectrum. Journal of School Psychology, 70, 64-73.

Aman, M. G., \& Singh, N. N. (1994). Aberrant Behavior Checklist Community. East Aurora NY Slosson: Supplementary manual.

American Psychiatric Association. (2013). Diagnostic and statistical manual of mental disorders: DSM-5 (5th ed.). Arlington, VA: American Psychiatric Association. Retrieved from https://search. library.wisc.edu/catalog/9911111397702121

Bal, V. H., Katz, T., Bishop, S. L., \& Krasileva, K. (2016). Understanding definitions of minimally verbal across instruments: Evidence for subgroups within minimally verbal children and adolescents with autism spectrum disorder. Journal of Child Psychology and Psychiatry, 57(12), 1424-1433.

Bearss, K., Taylor, C. A., Aman, M. G., Whittemore, R., Lecavalier, L., Miller, J., \& Scahill, L. (2015). Using qualitative methods to guide scale development for anxiety in youth with autism spectrum disorder. Autism, 20(6), 663-672.

Bland, J. M., \& Altman, D. G. (1995). Comparing methods of measurement: Why plotting difference against standard method is misleading. Lancet (London, England), 346(8982), 1085-1087.

Chandler, S., Howlin, P., Simonoff, E., O’Sullivan, T., Tseng, E., Kennedy, J., \& Baird, G. (2016). Emotional and behavioural problems 
in young children with autism spectrum disorder. Developmental Medicine and Child Neurology, 58(2), 202-208.

Charman, T., Palmer, M., Stringer, D., Hallett, V., Mueller, J., Romeo, R., Tarver, J., Perez, J. P., Breese, L., Hollett, M., Cawthorne, T., Boadu, J., Salazar, F., O’Leary, M., Beresford, B., Knapp, M., Slonims, V., Pickles, A., Scott, S., \& Simonoff, E. (2021). A novel group parenting intervention for emotional and behavioral difficulties in young autistic children: Autism spectrum treatment and resilience (ASTAR): A randomized controlled trial. Journal of the American Academy of Child and Adolescent Psychiatry, S0890-8567(21), 00298-00307.

De Los Reyes, A., Henry, D. B., Tolan, P. H., \& Wakschlag, L. S. (2009). Linking informant discrepancies to observed variations in young children's disruptive behavior. Journal of Abnormal Child Psychology, 37(5), 637-652.

De Los Reyes, A., Thomas, S. A., Goodman, K. L., \& Kundey, S. M. (2013). Principles underlying the use of multiple informants' reports. Annual Review of Clinical Psychology, 9, 123-149.

Einfeld, S. L., Ellis, L. A., \& Emerson, E. (2011). Comorbidity of intellectual disability and mental disorder in children and adolescents: A systematic review. Journal of Intellectual \&amp; Developmental Disability, 36(2), 137-143.

Estes, A., Olson, E., Sullivan, K., Greenson, J., Winter, J., Dawson, G., \& Munson, J. (2013). Parenting-related stress and psychological distress in mothers of toddlers with autism spectrum disorders. Brain \&amp; Development, 35(2), 133-138.

Flynn, S., Vereenooghe, L., Hastings, R. P., Adams, D., Cooper, S. A., Gore, N., \& Waite, J. (2017). Measurement tools for mental health problems and mental well-being in people with severe or profound intellectual disabilities: A systematic review. Clinical Psychology Review, 57, 32-44.

Fok, M., \& Bal, V. H. (2019). Differences in profiles of emotional behavioral problems across instruments in verbal versus minimally verbal children with autism spectrum disorder. Autism Research, 12(9), 1367-1375.

Granic, I., \& Patterson, G. R. (2006). Toward a comprehensive model of antisocial development: A dynamic systems approach. Psychological Review, 113(1), 101-131.

Hallett, V., Lecavalier, L., Sukhodolsky, D. G., Cipriano, N., Aman, M. G., McCracken, J. T., \& Scahill, L. (2013). Exploring the manifestations of anxiety in children with autism spectrum disorders. Journal of Autism and Developmental Disorders, 43(10), 2341-2352.

Harrison, P., \& Oakland, T. (2015). Adaptive Behavior Assessment System (ABAS-3) - Third edition Pearson

Jepsen, M. I., Gray, K. M., \& Taffe, J. R. (2012). Agreement in multiinformant assessment of behaviour and emotional problems and social functioning in adolescents with autistic and asperger's disorder. Research in Autism Spectrum Disorders, 6(3), 1091-1098.

Jones, R. R., Reid, J. B., \& Patterson, G. R. (1975). Naturalistic observation in clinical assessment. In P. McReynolds (Ed.), Advances in psychological assessment (pp. 42-95). Jossey-Bass.

Karver, M. S. (2006). Determinants of multiple informant agreement on child and adolescent behavior. Journal of Abnormal Child Psychology, 34(2), 251-262.

Kenny, L., Hattersley, C., Molins, B., Buckley, C., Povey, C., \& Pellicano, E. (2016). Which terms should be used to describe autism? Perspectives from the UK autism community. Autism, 20(4), 442-462.

Kent, R., \& Simonoff, E. (2017). Prevalence of anxiety in autism spectrum disorders. In C. M. Kerns, P. Renno, E. A. Storch, P. C. Kendall, \& J. J. Wood (Eds.), Anxiety in children and adolescents with autism spectrum disorder: Evidence-based assessment and treatment (pp. 5-32). Elsevier Inc.

Kerns, C. M., Kendall, P. C., Berry, L., Souders, M. C., Franklin, M. E., Schultz, R. T., \& Herrington, J. (2014). Traditional and atypical presentations of anxiety in youth with autism spectrum disorder. Journal of Autism and Developmental Disorders, 44(11), 2851-2861.

Lai, M. C., Kassee, C., Besney, R., Bonato, S., Hull, L., Mandy, W., \& Ameis, S. H. (2019). Prevalence of co-occurring mental health diagnoses in the autism population: A systematic review and meta-analysis. The Lancet. Psychiatry, 6(10), 819-829.

Langberg, J. M., Epstein, J. N., Simon, J. O., Loren, R. E. A., Arnold, L. E., Hechtman, L., \& Wigal, T. (2010). Parent agreement on ratings of children's attention deficit/hyperactivity disorder and broadband externalizing behaviors. Journal of Emotional and Behavioral Disorders, 18(1), 41-50.

Levinson, S., Neuspiel, J., Eisenhower, A., \& Blacher, J. (2021). Parent-Teacher disagreement on ratings of behavior problems in children with ASD: Associations with parental school involvement over time. Journal of Autism and Developmental Disorders, 51(6), 1966-1982.

Llanes, E., Blacher, J., Stavropoulos, K., \& Eisenhower, A. (2020). Parent and teacher reports of comorbid anxiety and ADHD symptoms in children with ASD. Journal of Autism and Developmental Disorders, 50(5), 1520-1531.

Lord, C., Rutter, M., DiLavore, P. C., Risi, S., Gotham, K., \& Bishop, S. (2012). Autism Diagnostic Observation Schedule (2nd ed.). Western Psychological Services.

Mash, E. J., \& Hunsley, J. (2005). Evidence-based assessment of child and adolescent disorders: Issues and challenges. Journal of Clinical Child and Adolescent Psychology, 34(3), 362-379.

Mason, D., McConachie, H., Garland, D., Petrou, A., Rodgers, J., \& Parr, J. R. (2018). Predictors of quality of life for autistic adults. Autism Research, 11(8), 1138-1147.

Mingins, J. E., Tarver, J., Waite, J., Jones, C., \& Surtees, A. D. R. (2020). Anxiety and intellectual functioning in autistic children: A systematic review and meta-analysis. Autism

Najman, J. M., Williams, G. M., Nikles, J., Spence, S., Bor, W., O'Callaghan, M., \& Andersen, M. J. (2000). Mothers' mental illness and child behavior problems: Cause-effect association or observation bias? Journal of the American Academy of Child and Adolescent Psychiatry, 39(5), 592-602.

National Institute for Health and Care Excellence. (2013). Antisocial behaviour and conduct disorders in children and young people: Recognition and management (CG158). United Kingdom: Author

National Institute for Health and Care Excellence. (2015) Challenging behaviour and learning disabilities: Prevention and interventions for people with learning disabilities whose behaviour challenges (NG11). United Kingdom: Author

National Institute for Health and Care Excellence. (2016). Autism spectrum disorder in adults: Diagnosis and management (CG142). United Kingdom: Author

Ng Fat, L., Scholes, S., Boniface, S., Mindell, J., \& Stewart-Brown, S. (2017). Evaluating and establishing national norms for mental wellbeing using the Short Warwick-Edinburgh Mental WellBeing Scale (SWEMWBS): Findings from the health survey for england. Quality of Life Research : An International Journal of Quality of Life Aspects of Treatment, Care and Rehabilitation, 26(5), 1129-1144.

NHS Health Scotland, University of Warwick and University of Edinburgh. (2008). Short Warwick Edinburgh Mental Well-Being Scale (SWEMWBS). United Kingdom: Author.

Palmer, M., Tarver, J., Paris Perez, J., Cawthorne, T., Romeo, R., Stringer, D., \& Charman, T. (2019). A novel group parenting intervention to reduce emotional and behavioural difficulties in young autistic children: Protocol for the autism spectrum treatment and resilience (ASTAR) pilot randomised controlled trial. British Medical Journal Open, 9, e029959. 
Palmer, M., Paris Perez, J., Tarver, J., Cawthorne Thomas, Frayne, M., Webb, S., Charman, T. (2020). Development of the observation schedule for children with autism - anxiety, behaviour and parenting (OSCA-ABP): A new measure of child and parenting behavior for use with young autistic children. Journal of Autism and Developmental Disorders

Podsakoff, P. M., MacKenzie, S. B., Lee, J., \& Podsakoff, N. P. (2003). Common method biases in behavioral research: A critical review of the literature and recommended remedies. Journal of Applied Psychology, 88(5), 879-903.

Reed, P., \& Osborne, L. A. (2013). The role of parenting stress in discrepancies between parent and teacher ratings of behavior problems in young children with autism spectrum disorder. Journal of Autism and Developmental Disorders, 43(2), 471-477.

Rescorla, L. A., Bochicchio, L., Achenbach, T. M., Ivanova, M. Y., Almqvist, F., Begovac, I., \& Verhulst, F. C. (2014). Parent-teacher agreement on children's problems in 21 societies. Journal of Clinical Child and Adolescent Psychology, 43(4), 627-642.

Royal College of Psychiatrists, British Psychological Society, \& Royal College of Speech and Language Therapists. (2007). Challenging behaviour: A unified approach (college report CR144). United Kingdom, Royal College of Psychiatrists

Salazar, F., Baird, G., Chandler, S., Tseng, E., O’Sullivan, T., Howlin, P., \& Simonoff, E. (2015). Co-occurring psychiatric disorders in preschool and elementary school-aged children with autism spectrum disorder. Journal of Autism and Developmental Disorders, 40(9), 1080-1093.

Schwartzman, J. M., Hardan, A. Y., \& Gengoux, G. W. (2021). Parenting stress in autism spectrum disorder may account for discrepancies in parent and clinician ratings of child functioning. Autism, $25,1601-1614$.

Silva, L. M., \& Schalock, M. (2012). Autism parenting stress index: Initial psychometric evidence. Journal of Autism and Developmental Disorders, 42(4), 566-574.

Simonoff, E., Pickles, A., Charman, T., Chandler, S., Loucas, T., \& Baird, G. (2008). Psychiatric disorders in children with autism spectrum disorders: Prevalence, comorbidity, and associated factors in a population-derived sample. Journal of the American Academy of Child and Adolescent Psychiatry, 47(8), 921-929.

Simonoff, E., Jones, C. R. G., Pickles, A., Happe, F., Baird, G., \& Charman, T. (2012). Severe mood problems in adolescents with autism spectrum disorder. Journal of Child Psychology and Psychiatry, 53(11), 1157-1166.

Simonoff, E., Jones, C. R., Baird, G., Pickles, A., Happe, F., \& Charman, T. (2013). The persistence and stability of psychiatric problems in adolescents with autism spectrum disorders. Journal of Child Psychology and Psychiatry, 54(2), 186-194.
Simonoff, E., Kent, R., Stringer, D., Lord, C., Briskman, J., Lukito, S., Baird, G. (2019). Trajectories in symptoms of autism and cognitive ability in autism from childhood to adult life: Findings from a longitudinal epidemiological cohort. Journal of the American Academy of Child and Adolescent Psychiatry

StataCorp. (2015). Stata statistical software: Release 14. StataCorp LP.

Stratis, E. A., \& Lecavalier, L. (2015). Informant agreement for youth with autism spectrum disorder or intellectual disability: A metaanalysis. Journal of Autism and Developmental Disorders, 45(4), 1026-1041.

Stratis, E. A., \& Lecavalier, L. (2017). Predictors of parent-teacher agreement in youth with autism spectrum disorder and their typically developing siblings. Journal of Autism and Developmental Disorders, 47(8), 2575-2585.

Stringer, D., Kent, R., Briskman, J., Lukito, S., Charman, T., Baird, G., Lord, C., Pickles, A., \& Simonoff, E. (2020). Trajectories of emotional and behavioral problems from childhood to early adult life. Autism, 24(4), 1011-1024.

Sukhodolsky, D. G., Lecavalier, L., Johnson, C., Smith, T., Swiezy, N., Bearss, K., Kalvin, C. B., \& Scahill, L. (2020). Anxiety in 3- to 7-year-old children with autism spectrum disorder seeking treatment for disruptive behavior. Autism, 24(2), 400-410.

Tarver, J., Pearson, E., Edwards, G., Shirazi, A., Potter, L., Malhi, P., \& Waite, J. (2021a). Anxiety in autistic individuals who speak few or no words: A qualitative study of parental experience and anxiety management. Autism, 25(2), 429-439.

Tarver, J., Vitoratou, S., Mastroianni, M., Heaney, N., Bennett, E., Gibbons, F., \& Santosh, P. (2021b). Development and psychometric properties of a new questionnaire to assess mental health and concerning behaviors in children and young people with autism spectrum disorder (ASD): The assessment of concerning behavior (ACB) scale. Journal of Autism and Developmental Disorders, 51(8), 2812-2828.

van der Oord, S., Prins, P. J., Oosterlaan, J., \& Emmelkamp, P. M. (2006). The association between parenting stress, depressed mood and informant agreement in ADHD and ODD. Behaviour Research and Therapy, 44(11), 1585-1595.

Yorke, I., White, P., Weston, A., Rafla, M., Charman, T., \& Simonoff, E. (2018). The association between emotional and behavioral problems in children with autism spectrum disorder and psychological distress in their parents: A systematic review and metaanalysis. Journal of Autism and Developmental Disorders, 48(10), 3393-3415.

Publisher's Note Springer Nature remains neutral with regard to jurisdictional claims in published maps and institutional affiliations. 